\title{
Spectral-domain optical coherence tomography of conjunctival mucosa-associated lymphoid tissue lymphoma with presumed choroidal involvement
}

\author{
Basil K. Williams Jr. • Irena Tsui • Tara A. McCannel
}

Received: 15 February 2010 /Revised: 23 May 2010 / Accepted: 24 May 2010 / Published online: 8 June 2010

(C) The Author(s) 2010. This article is published with open access at Springerlink.com

\begin{abstract}
Background Conjunctival mucosa-associated lymphoid tissue (MALT) lymphoma has been well-described, but rarely do these lesions demonstrate intraocular involvement. We report a case of conjunctival MALT lymphoma with intraocular involvement and novel spectral-domain ocular coherence tomography (SD-OCT) findings.

Patient and methods A 75-year-old woman with biopsyproven MALT lymphoma of the conjunctiva presented with ipsilateral yellowish diffuse choroidal infiltrates on fundoscopic examination. Choroidal involvement was documented clinically, on ultrasonography, on fluorescein angiography, and by SD-OCT. Treatment consisted of 3 weeks of oral doxycycline and six cycles of systemic chemotherapy with cyclophosphamide, vincristine, prednisone, and rituximab.

Results There was no evidence of progression or recurrence of MALT lymphoma after 9 months of follow-up. Despite complete resolution of clinical findings, ultrasound, and fluorescein angiography, the choroidal lesions remained unchanged on SD-OCT.
\end{abstract}

B. K. Williams Jr.

Rosalind Franklin University of Medicine and Science,

North Chicago, IL, USA

I. Tsui · T. A. McCannel

Retina Division and Ophthalmic Oncology Center,

Department of Ophthalmology, Jules Stein Eye Institute,

University of California,

Los Angeles, USA

T. A. McCannel $(\triangle)$

Jules Stein Eye Institute, University of California, Los Angeles,

100 Stein Plaza,

Los Angeles, CA 90095, USA

e-mail: TMcCannel@jsei.ucla.edu
Discussion Choroidal involvement of conjunctival MALT is rare, and can be successfully treated. Persistence of irregularities on SD-OCT did not influence management in the presence of clinical improvement and resolution on ultrasonography and fluorescein angiography.

Keywords Choroid - Lymphoma .

Mucosa-associated lymphoid tissue $\cdot$ MALT .

Optical coherence tomography $\cdot \mathrm{OCT}$

\section{Introduction}

Mucosa-associated lymphoid tissue (MALT) lymphoma is a low-grade variant of B-cell non-Hodgkins lymphoma that arises in extranodal tissue of the gastrointestinal tract (most frequently), lung, salivary gland, thyroid, orbit, and conjunctiva [1]. While localized conjunctival MALT lymphomas have been well-documented, there have only been three reports of intraocular extension [2-4]. These previous reports discuss symptoms, fundoscopic, and ultrasonographic findings, but do not include ocular coherence tomography (OCT). We present another atypical case of conjunctival MALT lymphoma, and discuss treatment considerations, spectral-domain (SD) OCT, and other ancillary studies in the presence of intraocular extension.

\section{Case report}

A 75-year-old woman began complaining of redness in the right eye 1 year prior to referral, which persisted despite both topical steroidal and non-steroidal therapy. During this period, she also developed increased intraocular pressure in the ipsilateral eye, which ultimately responded to treatment 
Fig. 1 a Early-phase

fluorescein angiogram of the right macula demonstrating mild hyperfluorescence

superotemporal to the optic disk. b Late-phase fluorescein angiogram of the right macula demonstrating diffuse choroidal staining throughout the macula. c B-scan ultrasonograpy of the right eye demonstrating diffuse low-reflective thickening posterior to the sclera, suggesting intraocular involvement
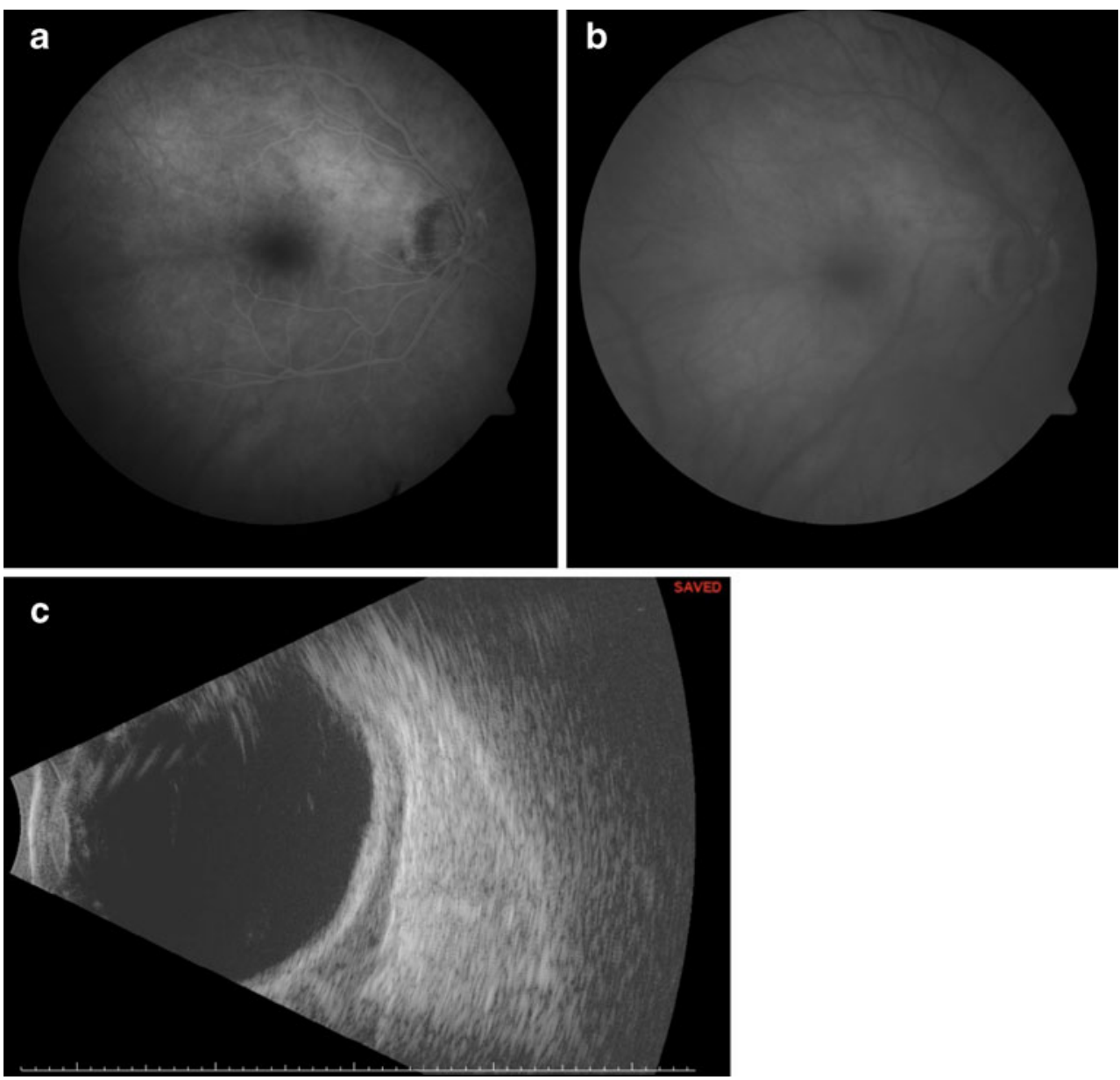

with fixed-combination brimonidine $0.2 \%$-timolol $0.5 \%$ twice a day. Her differential diagnosis included sarcoidosis, and systemic evaluation included chest X-ray, which demonstrated hilar lymphadenopathy. Conjunctival biopsy was performed to obtain a tissue diagnosis.

Pathology demonstrated small lymphocytic aggregates in the submucosa and lymphocytes containing eccentric nuclei with occasional Dutcher bodies and plasmacytoid appearance. Immunoperoxidase studies disclosed CD 10-negative cells, rare CD 3-positive, CD 5-positive, CD 23-positive, and CD43-positive cells, and a predominance of CD 20-positive cells. The diagnosis of low-grade conjunctival MALT lymphoma was made, and a complete systemic evaluation was obtained. Routine laboratory studies, computed tomography (CT) of the chest, abdomen, and pelvis, magnetic resonance imaging of the orbits, bilateral bone biopsies, serum $\mathrm{LDH}$, and $\beta_{2}$-microglobulin were all negative.

The patient was referred to the Ophthalmic Oncology Center at the Jules Stein Eye Institute for a second opinion. At her initial examination, best-corrected visual acuity was $20 / 20$ in the affected right eye and 20/125 in the left eye, which was amblyopic secondary to congenital esotropia.
The intraocular pressure was $14 \mathrm{mmHg}$ in both eyes. Slitlamp examination of the right eye revealed conjunctival hyperemia, a nasal pterygium, and trace cell in the anterior chamber. There were mild diffuse yellowish choroidal infiltrates on funduscopy of the right eye, and fluorescein angiography (Visupac, Carl Zeiss Meditec, Dublin, CA, USA) demonstrated corresponding late hyperfluoresence (Fig. 1a\&b). Ultrasonography (Eye Cubed, Ellex/Innovative Imaging, Minneapolis, MN, USA) displayed echolucent areas posterior to the sclera, with irregular borders consistent with an infiltrative choroidal process (Fig. 1c). SD-OCT (Cirrus HD-OCT, Carl Zeiss Meditec, Dublin, CA, USA) showed choroidal thickening in a lobular pattern in the right eye (Fig. 2a). The left eye had a normalappearing SD-OCT (Fig. 2b).

The patient underwent oral doxycyline $100 \mathrm{mg}$ twice a day for 3 weeks, in addition to six sessions of chemotherapy with cyclophosphamide, vincristine, prednisone (CVP), and rituximab. After 9 months of follow-up, the conjunctival hyperemia improved, and choroidal resolution was appreciated on fundoscopic examination, ultrasonography, and fluorescein angiography. However, SD-OCT remained 

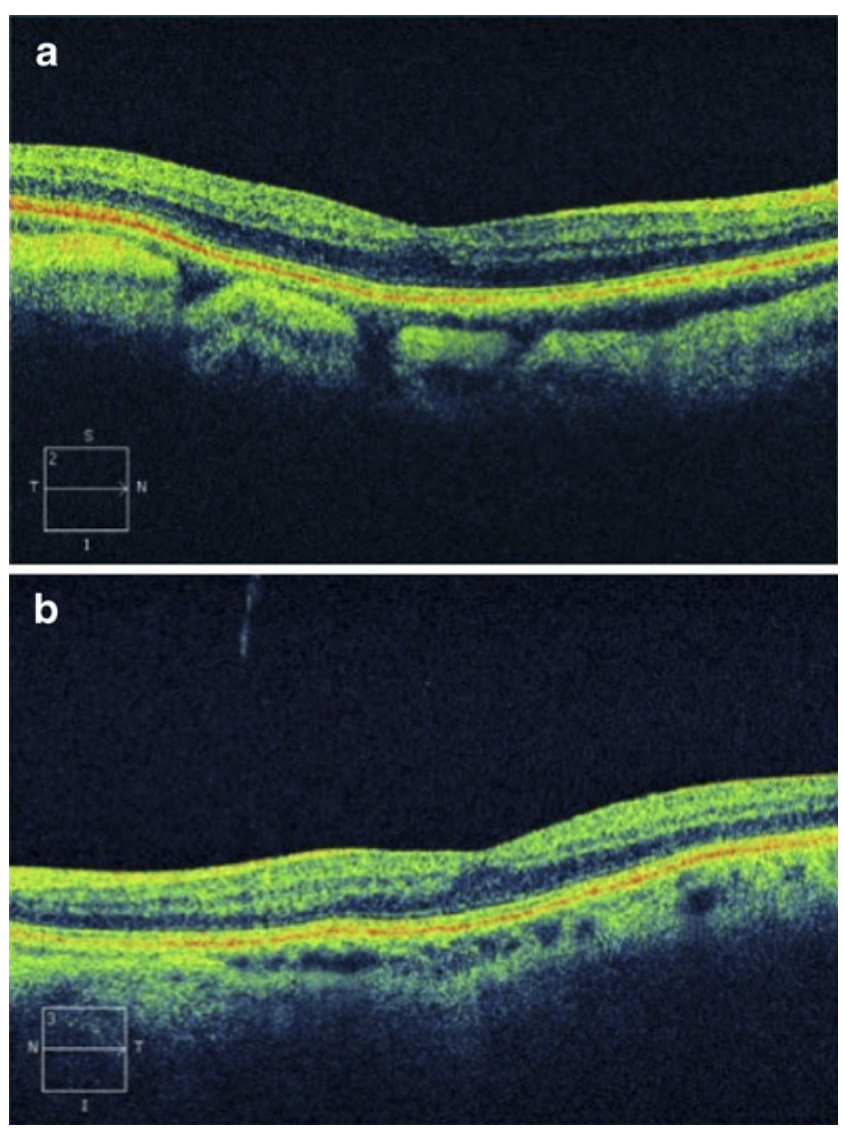

Fig. 2 a,b Spectral-domain optical coherence tomography scan of the (a) right eye and (b) left eye obtained at presentation to our center. a Large lobular masses and thickening of the choroid are demonstrated. b The left eye appeared normal

unchanged (Fig. 3). Maintenance therapy with Rituxamab was recommended for a total of 2 years.

\section{Discussion}

Medeiros and Harris [5] first described conjunctival MALT lymphoma in 1989, and these lesions have since been shown to comprise the majority of conjunctival lymphomas [6]. To date, three previous cases of conjunctival MALT lymphoma with presumed intraocular involvement have been reported [2-4]. Similar to our case, all three previous cases showed diffuse thickening of the posterior segment of the involved globe on ultrasonography. Two of the cases also described fundoscopic evidence of choroidal infiltrates, which were documented by fundus photography $[3,4]$. To our knowledge, this case is the first report of angiographic and SD-OCT documentation of choroidal involvement in MALT lymphoma.

In addition to providing images of the vitreomacular interface and retinal layers with high resolution, SD-OCT has also been used to visualize the choroid. Spaide et al. described enhanced depth imaging OCT of the choroid [7]. In this technique, the OCT machine was positioned closer to the eye, which created an inverted image and increased choroidal focus. Even in the absence of special techniques, SD-OCT has been used to visualize the choroid in albinism [8]. While our patient did not have ocular albinism, she did have a blonde fundus, which may have contributed to the ease of visualization of the choroid.

At the time of presentation, although her visual acuity was not affected, SD-OCT revealed lobular masses in the choroid, corroborating evidence of choroidal infiltrates. However, clinical improvement of both anterior and posterior findings of the lymphoma, ultrasound resolution, and angiographic improvement were noted after systemic treatment. Because the SD-OCT findings did not resolve in response to therapy, it is possible that the findings were not directly due to extension of the lymphoma. The origin of these lobular masses is unknown, but may represent congestion of the choroidal vasculature in response to the tumor or may be due to an unrelated process.

In addition to fluorescein angiography, which showed staining of the choroid at presentation that resolved with treatment, indocyanine green angiography may have been helpful to characterize the vascularity of the choroidal circulation better and help elucidate the pathogenesis of the choroidal infiltrate. As in the previous three cases, there is no pathological confirmation of tumor cells in the choroid in our case and choroidal extension is presumed.

In summary, this report is a rare case of presumed choroidal involvement secondary to MALT, which was responsive to chemotherapy. Abnormalities of the choroidal may persist on SD-OCT after treatment.

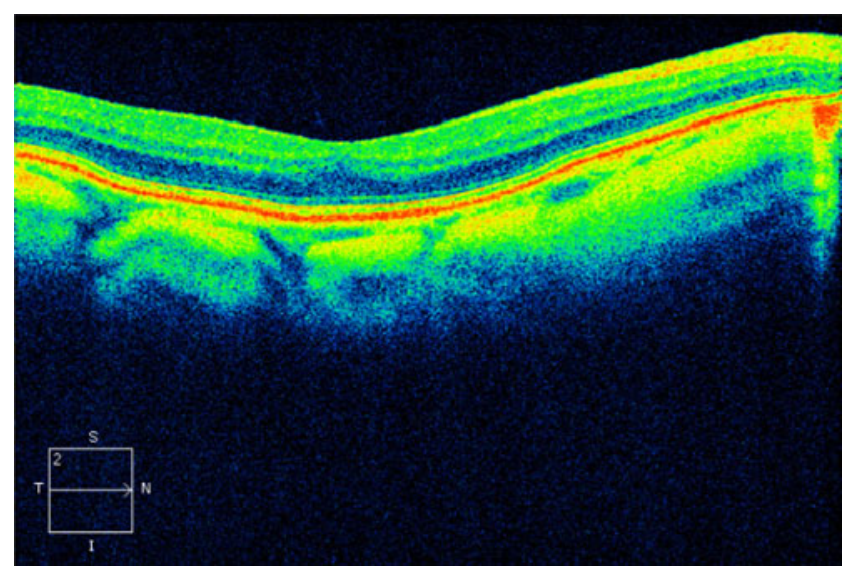

Fig. 3 Persistence of choroidal masses in the right eye on spectraldomain ocular coherence tomography scan, 9 months after treatment 
Disclosures The authors do not have any proprietary interest or potential conflicts of interest in the topics discussed. The authors have full control of all primary data and agree to allow Graefes Archive for Clinical and Experimental Ophthalmology to review our data upon request.

Open Access This article is distributed under the terms of the Creative Commons Attribution Noncommercial License which permits any noncommercial use, distribution, and reproduction in any medium, provided the original author(s) and source are credited.

\section{References}

1. Wotherspoon AC, Hardman-Lea S, Isaacson PG (1994) Mucosaassociated lymphoid tissue (MALT) in the human conjunctiva. J Pathol 174:33-37
2. Hoang-Xuan T, Bodaghi B, Toublanc M et al (1996) Scleritis and mucosa-associated lymphoid tissue lymphoma: a new masquerade syndroma. Ophthalmology 103:631-635

3. Sarraf D, Jain A, Dubovy A et al (2005) Mucosa-associated lymphoid tissue lymphoma with intraocular involvement. Retina 25:94-98

4. Ramulu P, Iliff NT, Green RW, Kuo IC (2007) Asymptomatic conjunctival mucosa-associated lymphoid tissue-type lymphoma with presumed intraocular involvement. Cornea 26:484-486

5. Medeiros LJ, Harris NJ (1989) Lymphoid infiltrates of the orbit and conjunctiva. A morphologic and immunophenotypic study of 99 cases. Am J Surg Pathol 13:459-471

6. Stefanovic A, Lossos IS (2009) Extranodal marginal zone lymphoma of the ocular adnexa. Blood 114:501-510

7. Spaide RF, Koizumi H, Pozonni MC (2008) Enhanced depth imaging spectral-domain optical coherence tomography. Am J Ophthalmol 146:496-500

8. Seo JH, Yu YS, Kim JH et al (2007) Correlation of visual acuity with foveal hypoplasia grading by optical coherence tomography in albinism. Ophthalmology 114:1547-1551 